\title{
Benefits of Exercise with Mini Tennis in Intellectual Disabilities: Effects on Body Image and Psychopathology
}

\author{
Maria Carolina Hardoy ${ }^{1,2, *}$, Maria Luisa Seruis ${ }^{2}$, Francesca Floris ${ }^{2}$, Federica Sancassiani ${ }^{2}$, Maria \\ Francesca Moro ${ }^{2}$, Gisa Mellino ${ }^{2}$, Maria Efisia Lecca ${ }^{2}$, Siria Adamo², Mauro Giovanni Carta ${ }^{2}$ \\ ${ }^{1}$ Reald University Vlore, Albania; ${ }^{2}$ Center for Liaison Psychiatry and Psychosomatics, University of Cagliari, Italy
}

\begin{abstract}
The present study is aimed at evaluating the efficacy of an introductory mini tennis programme as a therapeutic aid in the psychosocial rehabilitation of participants affected by mild/moderate intellectual disability in semi-residential care.

Two groups ( $\mathrm{N}=12)$ of participants diagnosed with intellectual disability, one of which followed the mini tennis rehabilitation programme, were compared at time t0, t1 (after 2 months) and t 2 (after 6 months).

Psychopathological status was assessed by means of the Italian version of the Assessment and Information Rating Profile (AIRP). Motor coordination, lateral dominance and body scheme were assessed by means of structured tools.

Psychopathological total scores showed a statistically significant decrease in the experimental group in comparison with the control group. A statistical decrease in the group with the mini tennis rehabilitation programme was found also in the anxiety sub-scale while the sub-scales schizophrenia, depression, adjustment disorder, personality problems, somatoform disorders and psychosexual disorders did not reach any statistical difference between groups.

A statistically significant increase in the visuo manual coordination was highlighted in the experimental versus the control group. No statistically significant differences were reported with regard to general movement skills, dynamic balance and coordination.

In spite of the limitations of this study, the results obtained are encouraging and suggest the potential efficacy of mini tennis as an auxiliary aid in rehabilitation programmes, particularly to improve visuo manual coordination skills and to boost the patient/participants' self esteem. These findings warrant confirmation by further research studies.
\end{abstract}

Keywords: Intellectual disability, mini tennis, anxiety disorders, motor disabilities, rehabilitation, Assessment and Information Rating Profile (AIRP)

\section{INTRODUCTION}

The present study is aimed at evaluating the efficacy of an introductory mini tennis programme as a therapeutic aid in the psychosocial rehabilitation of participants affected by mild-moderate intellectual disability (ID) in semi-residential care.

The initial hypothesis was that the introduction of a structured activity of pre-competitive sport training, within a complex psychosocial rehabilitative plan, could have exerted a positive influence on:

1. Psychomotor parameters related to paresis and coordination problems which often accompany intellectual disability, by integrating the personalised psychomotor treatment plan devised in the rehabilitative protocol; participants in care often show problems of stabilisation in laterality and crossed laterality; the attainable goal was to achieve, through practising sport, a stabilisation of laterality so as to improve awareness of spatial notions $[1,2]$.

*Address correspondence to this author at the Center for Liaison Psychiatry and Psychosomatics, University of Cagliari, Italy; Tel: +39 335499994; Fax: +39 070 6093498; E-mail: mgcarta@tiscali.it.
2. Psychosocial skills by providing a stimulus towards socialisation and improving self-esteem. [3-5]. An opportunity such as that provided by the mini tennis programme could accordingly elicit an improvement in their social relationships and support their motivation; the latter may indeed influence and sustain the entire rehabilitative project thus improving patient/participants' spatial skills. Moreover sport activity increases mood and physical quality of life [6].

\section{METHODOLOGY}

\section{Setting}

The study was carried out in three "semi-residential daily rehabilitation centres" in which people whit ID stay for 6 hours (from $8 \mathrm{AM}$ to $2 \mathrm{PM}$ ) six days each week attending occupational / rehabilitation programs including occupational therapy (carpentry, earthenware, needled), sport activities (only soccer before mini tennis introduction) and physiotherapy (when needed). A total of 82 people with ID was in charge at the three centres at the time of the study.

\section{Study Design and Sample}

The study is a clinical controlled not randomised trial. Two groups of 12 participants each (experimental and control group), were selected for the study. 
Table 1. Study Sample

\begin{tabular}{|c|c|c|c|c|}
\hline & Number & Sex & Age* & ID Score\# \\
\hline \hline Experimental Group & 12 & $5 \mathrm{M}$ & $27.6 \pm 6.7$ & $52.3 \pm 3.4$ \\
\hline Control Group & 12 & $7 \mathrm{M}$ & $26.9 \pm 10.2$ & $51.8 \pm 3.7$ \\
\hline
\end{tabular}

*DF, $1,23,23 ; \mathrm{F}=0.1 ; \mathrm{P}=0.84$

\#DF,1,23,23; $\mathrm{F}=0.85 ; \mathrm{P}=0.35$

Inclusion criteria were:

ID mild - moderate measured with Wechsler Adult Intelligence Revised in the Italian version [7].

Both sex, aged 18-40.

Manifest interest / agreement to take part in the physical activities.

Exclusion criteria were:

Any contraindications to physical activity.

Diagnosis of severe neurological and orthopedic disorders at the time of the study (Multiple Sclerosis, Rheumatoid Arthritis, Amyotrophic Lateral Sclerosis, Stroke, radicular or medullar compression, joint fractures, joint surgery, acquired limitations of joint movement).

The two groups were divided according to whether or not participants preferred to be enrolled in the introductory structured programme of mini tennis (experimental group) or playing football (soccer in USA) games (without rigid rules). The football games were played at the same time that the other group was receiving the mini tennis course.

Study sample with age, sex and ID score is shown in Table 1. The two groups were homogeneous with regard to both personal and social data and parameters of clinical evaluation; no differences were found as to their motor disabilities or nature of the psychopathological disorder.

All participants (in the experimental and in the control group) underwent standard evaluation with the study tools at the beginning of the programme (t0), after two months (t1) and at the end (after 6 months) of the mini tennis programme (t2).

Participation in the study was voluntary and informed consent was obtained with participants or with tutor if necessary. Participants and tutors were informed that they could withdraw their consent to participate at any time, with no negative consequences on their future treatment.

\section{Description of Mini Tennis}

The mini tennis is a modified form of tennis which has been adapted for young children and the disabled. It is played using lightweight soft balls and short-handled lightweight racquets.

Mini tennis is played in a court half the size of a normal tennis court. Younger start playing with soft, large ball then move up to modified tennis balls. Racquets start off with a small 19" frame before increasing to a full size junior racket. The kind of racket should be adapted to the player to ensure that the size and weight is appropriate for the players' strength.
The scoring system features shorter games and tie-breaks before embracing regular scoring. Balls come in four different colours. Red, orange, and green are for mini tennis and are low compression to make learning the correct technique possible.

The mini tennis programme was carried out over a 6 month-period with 3-hour sessions held twice weekly (one of which included an actual game of mini tennis); meetings were attended in sub-groups of 6 participants per group. Each session was conducted by a federal tennis teacher and by an athletic trainer. The programme was divided into three phases although no strict distinction was imposed: objectives and techniques of the three phases often partially overlapped during the same session. The objective to be reached during the first month of meetings regarded the first two phases alone, which were the main goal also during the second month of meetings. Over the following months concepts and exercises related to these preliminary phases were regularly recalled once the third phase themes had become prominent.

1st phase: exercises to familiarise participants with equipment: ball, wooden paddles, racquet.

2nd phase: development of coordination skills: visuomanual, general dynamic and temporospatial skills.

3rd phase: learning of the basic tennis techniques.

All training sessions were scheduled in a playful manner.

\section{Evaluation Instruments and Study Tools}

The following evaluation instruments were administered:

Assessment and Information Rating Profile (AIRP) [8] in its Italian version [9]: this instrument provides a complete psychopathological profile and has been purposely studied for the evaluation of mental, psychopathological and behavioural conditions in people with intellectual disabilities. Some authors of the present study had contributed to the task of validating the latter assessment tool. The AIRP in the Italian version showed a good test-retest reliability and was already used in clinical studies [10].

The Bruininks-Oseretsky Test of Motor Proficiency adapted for young adults [11] was used to assess general motor ability profile: evaluation of the psychomotor profile through variables related to psychomotor age was performed for the following areas: visuo manual coordination, general movement skills, dynamic balance and coordination. Scores were given in terms of age-skills (from 0 to 11).

The perception of the body scheme was measured by means of Vayer's Body Scheme Test adapted by Pisaturo [12] with a final score ranging from 1 to 4 . 
Table 2. AIRP: Total Score (Mean \pm SD)

\begin{tabular}{|c|c|c|c|}
\hline & T0 & T1 & T2 \\
\hline Experimental group & $6.5 \pm 4.3$ & $5.9 \pm 4.2$ & $5.3 \pm 3.7$ \\
\hline Control group & $5.8 \pm 3.7$ & $5.7 \pm 3.9$ & $5.9 \pm 4.3$ \\
\hline
\end{tabular}

$\mathrm{F}=8,2, \mathrm{P}<0,05$ (MANOVA DF 2,22)

Table 3. AIRP: Anxiety Score (Mean \pm SD)

\begin{tabular}{|c|c|c|c|}
\hline & T0 & T1 & T2 \\
\hline \hline Experimental group & $3.2 \pm 1.8$ & $2.8 \pm 2.0$ & $2.3 \pm 1.3$ \\
\hline Control group & $2.8 \pm 2.3$ & $2.7 \pm 2.1$ & $2.8 \pm 2.3$ \\
\hline
\end{tabular}

$\mathrm{F}=12,1, \mathrm{p}<0,01$ MANOVA $(\mathrm{DF} 2,22)$

The degree of lateral dominance was also assessed, with Harris Test of Lateral Dominance [13]: items 2, 8 and 9 selected by Vayer [14].

\section{Statistical Analysis}

Statistical analysis results relating to the influence of mini tennis programme on psychological symptoms, on maladjusted behaviours and self-esteem values were analysed by assessing the findings obtained in both experimental and control groups with AIRP at times $t 0, t 1, t 2$. Data obtained were studied using multivariate analysis of variance for repeated measurements (MANOVA) through the computer program SPSS. The same protocol was used to analyse results of the motor coordination test. Results of the Vayer's Body Scheme Test were examined by means of the Friedman's rank analysis of variance whilst findings from the Harris Test of Lateral Dominance were evaluated using the Fisher exact test ( $\mathrm{t} 0$ vs $\mathrm{t} 2$ ).

\section{RESULTS}

\section{Psychopathological Symptoms}

As shown in Table 2, the total score obtained revealed a satisfactory decrease in the experimental group with statistically significant differences compared to the control group; differences are mainly related to the sub-scale anxiety (as in Table 3) while no statistically significant differences between the two groups were observed for the sub-scales schizophrenia, depression, adjustment disorder, personality problems, somatoform disorders and psychosexual disorders.

\section{Psychomotor Profile}

Visuo manual coordination increased in the experimental group from $7.6 \pm 0.8$ to $8.2 \pm 1.0$, while scores for the control group were $8.3 \pm 2.0$ to $8.2 \pm 1.2$. This difference reached a statistical significance $(\mathrm{F}=6,5 ; \mathrm{p}<0,05$ MANOVA DF 2,22). No statistically significant differences were found with regard to general movement skills, dynamic balance and coordination.

Vayer's Body Scheme Test, provided the following scores: Experimental group: T0 3 points (15\%), 2 points $(85 \%)$. T2 3 points $(60 \%), 2$ points $(40 \%)$ (chi square=3,9; $\mathrm{p}<0,05$, analysis of variance according to Friedman's ranks). Control group: T0 3 points $(60 \%), 2$ points $(40 \%)$. T2 3 points $(60 \%), 2$ points $(40 \%)$ (no statistical difference).
Findings of the Lateral Dominance (items 2, 8 and 9 selected by Vayer), revealed a reinforcement of laterality in two participants from the experimental group (16.7\%), none of the control group showed any reinforcement of the laterality (no significant difference at Fisher exact text).

\section{DISCUSSION}

Due to the fact that also football needs, obviously, a physical activity as mini tennis, the improvement in anxiety and in the total AIRP score do not have to be interpreted as a consequence of the exercise itself. In order to fully comprehend the significance of this finding, it should first be underlined that until the introduction of the mini tennis in the rehabilitation programme, the only sport practised was football. However, several participants had taken part in this activity with caution as they considered this sport too difficult and at times problems were caused by the ensuing physical contact; probably this activity was subsequently a source of frustration and repercussions were not only limited to the sport itself. The marked improvement in anxiety symptoms (not detected in the control group) may represent the consequence of an increased self-confidence of an activity less frustrating than football.

Considering the above premises and the novelty of the programme, it is easy to see the reason why this type of activity was so well accepted.

An alternative explanation of the improvement in general psychopathology and in anxiety in the experimental group (and not in the control group), may be related to the positive effect on psychopathology of the improvement in the body perception. A large literature reported the difficulty in severe psychopathological conditions such as schizophrenia, autism, anorexia nervosa, to create a "theory of mind" and to develop social cognitions $[15,16]$. Social cognition refers to how people conceive, perceive, and draw inferences about mental and emotional states of others in the social world. Recently the literature underlined the relationship between skills in social cognition and the ability in representation and imagination the others [17]. In this perspective a paper indicated that in the imagination of a person performing an action exists two distinct biases: a perceptual-mnemonic bias due to subjects' visual experience of others' actions and a motor bias due to subjects' experience of self-made actions, 
encouraging them to imagine movements performed by other [18]. In the imagination and the representation of others the self perception is relevant. The self perception may be also relevant in improving the social cognition skill and the psychopathology. Nevertheless, this may only be considered as an heuristic hypothesis.

The improvement reported in the performance of the psychomotor profile tests, together with the decreased anxiety and the changes in general psychopathology, are findings of considerable importance; a similar improvement in performance may represent the psychosomatic synthesis of a global improvement in self-perception.

In this way, an explanatory hypothesis may be that a structured programme with bi-laterality of exercise, repetition of task not well performed but in a strong motivating setting may exert really a valid tool to improve body perception and self esteem.

However, several "structural" limits which may have considerably influenced the development of the programme and its final evaluation were present from the outset. Firstly, the advanced age of participants and the presence of wellestablished motor schemes learnt through long-term repetition of gestures; the training required by the mini tennis programme was not sufficient to modify these patterns. Secondly, the limited resources available for the development of the programme, together with the small number of participants and the frequency of sessions.

In spite of these limitations however, the results obtained in the study are definitely encouraging and suggest the potential efficacy of mini tennis as an auxiliary aid in rehabilitation programmes, particularly to improve visuo manual coordination skills and to boost the participants' self esteem. The findings obtained warrant confirmation by further research studies.

\section{REFERENCES}

[1] Carlier M, Desplanches AG, Philip N, et al. Laterality preference and cognition: cross-syndrome comparison of patients with trisomy 21 (Down), del7q11.23 (Williams-Beuren) and del22q11.2 (Di-
George or Velo-Cardio-Facial) syndromes. Behav Genet 2011; 41 (3): 413-22.

[2] Leconte P, Fagard J. Lateral preferences in children with intellectual deficiency of idiopathic origin. Dev Psychobiol 2006; 48(6): 492-500.

[3] Stanish HI, Frey GC. Promotion of physical activity in individuals with intellectual disability. Salud Publica Mex 2008; 50 (Suppl 2): s178-84.

[4] Frey GC, Stanish HI, Temple VA. Physical activity of youth with intellectual disability: review and research agenda (Review). Adapt Phys Activ Q 2008; 25(2): 95-117.

[5] Pilu A, Sorba M, Hardoy MC, et al. Efficacy of physical activity in the adjunctive treatment of major depressive disorders: preliminary results. Clin Pract Epidemiol Ment Health 2007; 3: 8.

[6] Carta MG, Hardoy MC, Pilu A, et al. Improving physical quality of life with group physical activity in the adjunctive treatment of major depressive disorder. Clin Pract Epidemol Ment Health 2008; 4(1): 1 .

[7] Lang M, Michelotti C. Le Scale Wechsler per Adulti. Valutazione delle Funzioni Cognitive e Analisi del Processo. Milano: Raffaello Cortina 2008.

[8] Bouras N, Drummond C. Community psychiatric service in mental handicap: six years of experience. London: NUPRD 1989.

[9] Carta MG, Hardoy MC. Assessment and Information Rating Profile. Italian version. Cagliari: CUEC 1998.

[10] Carta MG, Hardoy MC, Dessì I, Hardoy MJ, Carpiniello B. Adjunctive gabapentin in patients with intellectual disability and bipolar spectrum disorders. J Intellect Disability Res 2001; 45: 139-45.

[11] Myrelid A, Bergman S, Elfvik Strömberg M, et al. Late effects of early growth hormone treatment in Down syndrome. Acta Paediatr 2010; 99(5): 763-9.

[12] Pisaturo C. Appunti di psicomotricità. Lo schema corporeo in psicomotricità. Roma: Piccin-Nuova Libraria 1996.

[13] Harrys AJ. Harris test of lateral dominance. Manual of directions for administration and interpretation. $3^{\text {rd }}$ ed. New York: The Psychological Corporation 1958.

[14] Vayer P. Exercice rythmé et organisation du temps. Annales de Rèeducation Phisique 1982; 88: 92-106.

[15] Brüne M, Ozgürdal S, Ansorge N, et al. An fMRI study of "theory of mind" in at-risk states of psychosis: comparison with manifest schizophrenia and healthy controls. Neuroimage 2011; 55 (1): 32937.

[16] Gál Z, Egyed K, Pászthy B, Németh D. Impaired theory of mind in anorexia nervosa. Psychiatr Hung 2011; 26(1): 12-25.

[17] Jarrold C, Mansergh R, Whiting C. The representational status of pretence: evidence from typical development and autism. Br J Dev Psychol 2010; 28(Pt 2): 239-54.

[18] Marzoli D, Mitaritonna A, Moretto F, Carluccio P, Tommasi L. The handedness of imagined bodies in action and the role of perspective taking. Brain $\operatorname{Cogn} 2011 ; 75(1): 51-9$.

Received: April 28, 2011
Revised: June 20,2011

work is properly cited. 